\title{
Application of Pharmacokinetics/Pharmacodynamics (PK/PD) in Designing Effective Antibiotic Treatment Regimens
}

\author{
Ghada F. Ahmed ${ }^{1}$ and Ayman M. Noreddin',** \\ 1 University of Minnesota \\ ${ }^{2}$ Hampton University \\ USA
}

\section{Introduction}

Designing antibiotic dosing regimens is often not optimal and the dose-response relationship for most antibiotics is not well-known ${ }^{1}$. Both Pharmacokinetics (PK) and Pharmacodynamics (PD) are characteristics of antimicrobial agents that should be considered in the development of effective antibiotic therapy. By linking the concentration time profile at the site of action to the drug effect (PK/PD), the effect of varying dosage regimens against pathogens could be simulated enabling the identification of effective dosage strategies. It is known that inadequate antibiotic dosing could not only lead to a therapeutic failure, but also to the development of bacterial resistance. Importantly, the evolution of resistance in pathogenic bacteria combined with the decreasing interest from the pharmaceutical industry in developing new antibiotics has created a major public health problem $^{3}$. Therefore, the activities to maintain the effects of existing antibiotics and prolong their useful life span have a high priority.

PK/PD analysis proved to be a useful tool for investigating effective therapies that minimize the emergence of antibiotic tolerance. Although the European Medicine Agency and FDA clearly recommends PK/PD assessment for new compounds, there are no standardized procedures for such analyses for antibiotics ${ }^{3}$. For characterizing the PD of an antibiotic, bacterial growth and death under antibiotic exposure have to be investigated. Since these are difficult to measure in human tissues, animal and in vitro models have been developed.

Although animal models provide similar growth conditions for bacteria and imitate the characteristics of human infection, they exhibit different PK and drug disposition profiles compared to humans ${ }^{4}$. In contrast, in vitro models have the advantage of simulating human PK and bacterial resistance analysis. Therefore, they are considered adequate for the investigation of antimicrobial activity ${ }^{4}$.

Frequently, the pharmacodynmic relationship is reduced to a single parameter, the Minimum inhibitory concentration (MIC), even though antibiotics with the same MIC can

${ }^{*}$ Corresponding Author 
have different PD functions ${ }^{1}$. Differences in the kill profiles exhibited by antibiotics is hypothesized to be influenced by disparities of their PD characteristics that- if not taken into account during the design of treatment regimens- may lead to therapeutic failure and development of resistance.

In this study, five antibiotics of five different classes were considered for PK/PD analysis. The antibiotics were Ciprofloxacin (a fluorquinolone that acts by inhibiting bacterial DNA gyrase enzyme), Ampicillin (a beta-lactam antibiotic acts by inhibiting bacterial cell wall synthesis), and finally Rifampin, Streptomycin, and Tetracycline (protein synthesis inhibitors). The antibiotics were further classified according to their pharmacodyamic properties into concentration dependent antibiotics (Ciprofloxacin, Rifampin and Streptomycin), and time dependent antibiotics (Ampicillin and Tetracycline).

PK/PD simulations using STELLA ${ }^{\circledR}$ (Version 9.1, isee system inc, NH, USA) were used to investigate the effect of various PD parameters on the achievement of the therapeutic outcome and in designing effective dosing regimen that help overcome the development of resistance to the antibiotic.

\section{Pharmacokinetic analysis}

The PK of the antibiotics was assumed to follow one compartment disposition characteristics with a first order elimination rate constant. The drug was given in a dose that gave an initial plasma concentration equivalent to 5 times the MIC and the plasma concentration was allowed to decrease mono-exponentially to a minimum of 0.5 times the MIC over 8 hours according to the following equation

$$
\mathrm{C}_{\mathrm{t}}=\mathrm{C}_{0} \exp \{-\mathrm{kt}\}
$$

Where $C_{t}$ is the plasma concentration at time $t, C_{0}$ is the initial plasma concentration and $k$ is the first-order elimination rate constant.

Doses were administered as multiple IV boluses with a time interval of 8 hours. The system was assumed to be at a steady state. The volume of distribution was assumed to be fixed (equals 1); since the data were obtained from an in vitro experiment. Clearance $\left(\mathrm{k}^{*} \mathrm{v}\right)$ was then assumed to be equivalent to the value of the elimination rate constant $\mathrm{k}$. For this analysis, Ciprofloxacin, Ampicillin, Rifampin, Streptomycin and Tetracycline were the considered antibiotics.

\section{Pharmacodynamic analysis}

\subsection{Effect of exposure to antibiotics on the bacterial net growth rate}

The net growth rate under certain antibiotic pressure could be described by the following equation:-

$$
\Psi(a)=\Psi \max -\mu(a)
$$

Where is the net growth rate at antibiotic concentration a, is the growth rate of the bacteria in absence of antibiotic, and is the death rate of the bacterial population exposed to an antibiotic concentration a. Therefore, the net growth rate of the bacterial population under antibiotic treatment could be described by the following model:- 


$$
\Psi(a)=\Psi \max -\frac{(\Psi \max -\Psi \min ) \times\left(\frac{a}{z M I C}\right)^{k}}{\left(\frac{a}{z M I C}\right)^{k}-\frac{\Psi \min }{\Psi \max }}
$$

Whereis the minimum net growth rate of the bacteria at high antibiotic concentration, $a$ is the antibiotic concentration, zMIC is the pharmacodynamic MIC (concentration of the antibiotic at which the net bacterial growth rate was calculated to be zero), and $\mathrm{K}$ is the Hill coefficient which is a measure of the steepness of the sigmoidal relationship between antibiotic concentration and the net growth rate.

The model was constructed to evaluate the effect of the antibiotics against a single microorganism (E. coli); and therefore, the maximum growth rate is assumed to be constant.

The minimum growth rate, zMIC as well as the Hill coefficient will, however, change due to different antibiotic treatments. Bacterial net growth rate against the antibiotic concentration was analyzed for the aforementioned antibiotics.

\section{PK/PD analyses}

The bacterial population was modeled during the mono-exponential growth phase. The change in bacterial density was modeled according to the following equation:-

$$
\mathrm{dx} / \mathrm{dt}=\text { growth rate }- \text { kill rate due to antibiotic }
$$

Where $x$ is the bacterial density measures in colony forming units per $\mathrm{mL}(\mathrm{CFU} / \mathrm{mL})$

Time plots of the logarithm to the base 10 of the bacterial density were generated and the effect of the PD parameters (MIC, K, minimum growth rate, and maximum growth rate) on the achievement of the target treatment outcome was investigated through a sensitivity analysis in STELLA ${ }^{\circledR}$. The target treatment outcome was defined as three $\log _{10}$ decrease in the bacterial density over a 24 hour course of the treatment.

\subsection{Design of antibiotics treatment strategies}

Antibiotics are generally classified to concentration dependent and time dependent agents. In the current analysis, treatment strategies were constructed and tested under different scenarios. Results from the simulation were used to conclude on the most appropriate treatment option for different classes of antibiotics.

Increasing the MIC by two fold and four folds supported the simulation of bacterial resistance against the antibiotic treatments. The outcome target of the treatment is to achieve three $\log _{10}$ decrease in the bacterial density over a 24 hours course of treatment through the given dosage regimen. Towards that target, two treatment strategies were used. The first was to increase the area under the antibiotic concentration time curve above the minimum inhibitory concentration (AUC>MIC) through increasing the administered dose, while the second was to increase the percentage of time during which the drug concentration is above the MIC relative to the dosing interval ( $\% t>M I C)$ through decreasing the dosing interval with smaller maintenance doses. Both scenarios were 
applied to each of the investigated antibiotics and the outcomes of the treatment regimens were compared.

\section{Results}

\subsection{The pharmacokinetic model}

The disposition of the antibiotics was shown to follow a one compartment model with a first order elimination rate. The achievement of the steady state was assumed form the beginning of therapy. (Figure 1a and 1b)

The antibiotics were given as multiple intravenous boluses every 8 hours over a course of 24 hours.

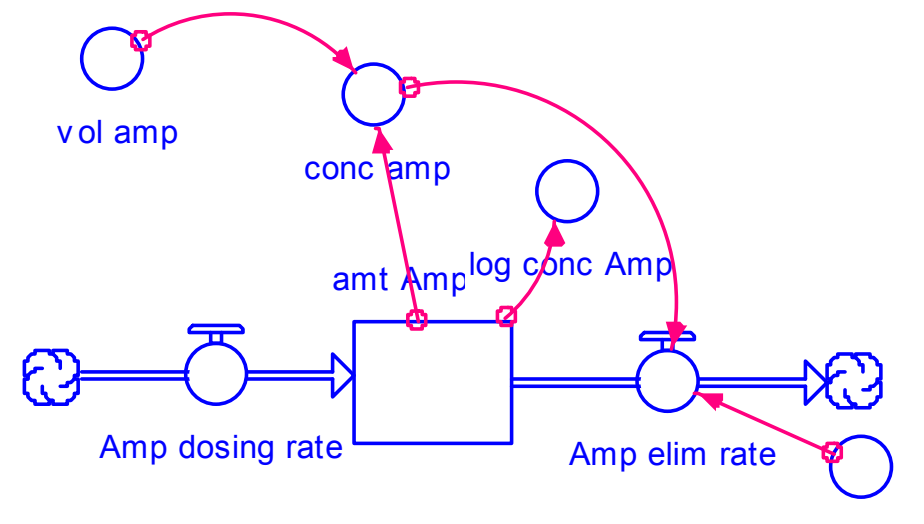

cl amp

Fig. 1a. STELLA ${ }^{\circledR}$ model simulating the one compartment PK model with first order elimination rate constant for Ampicillin

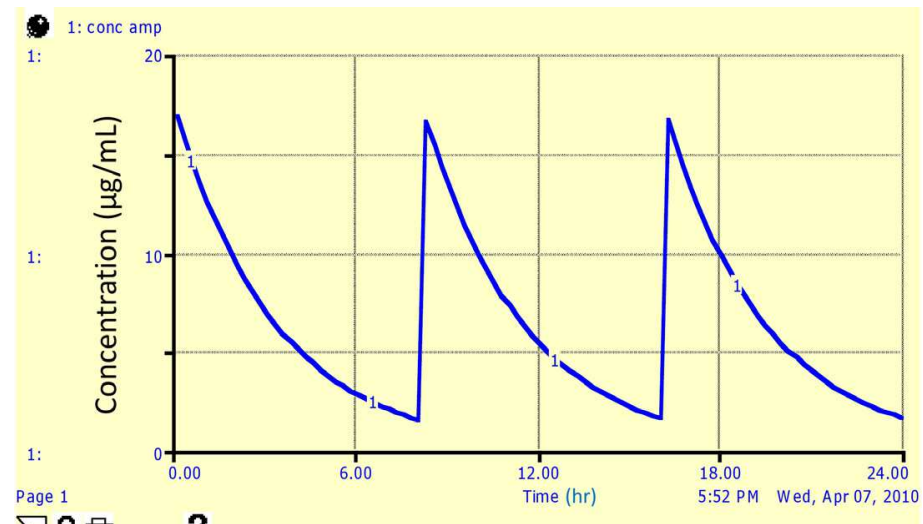

$\nabla 6$ ? ?

Fig. 1b. Simulation of the Ampicillin steady state concentration time profile form the PK model. 


\subsection{Pharmacodynamic analysis}

\subsubsection{Effect of antibiotic concentration on the bacterial net growth rate}

Changing antibiotic concentration is assumed to be affecting the net growth rate of the bacteria cultured in the in vitro system. The infection was therefore assumed to occur in the central compartment. The pharmacodynamic parameter estimates for the five considered antibiotics are displayed in Table 1. STELLA ${ }^{\circledR}$ model (Figure 2) was created to determine the effect of changing drug concentration on the net bacterial growth rate according the pharmacodynamic function expressed in equation 3.

\begin{tabular}{ccccc}
\hline $\begin{array}{c}\text { PD parameter/ } \\
\text { drug }\end{array}$ & $\begin{array}{c}\text { Max. growth } \\
\text { rate }\left(\mathrm{h}^{-1}\right)\end{array}$ & $\begin{array}{c}\text { Min. growth } \\
\text { rate }\left(\mathrm{h}^{-1}\right)\end{array}$ & $\mathrm{K}$ & zMIC $(\mu \mathrm{g} / \mathrm{mL})$ \\
\hline Ciprofloxacin & 0.75 & -6.5 & 1.1 & 0.017 \\
Ampicillin & 0.75 & -4.0 & 0.75 & 3.4 \\
Rifampin & 0.75 & -4.3 & 2.5 & 12.0 \\
Streptomycin & 0.75 & -8.8 & 1.9 & 18.5 \\
Tetracycline & 0.75 & -8.1 & 0.61 & 0.67 \\
\hline
\end{tabular}

Table 1. Pharmacodynamic parameter estimates ${ }^{1}$

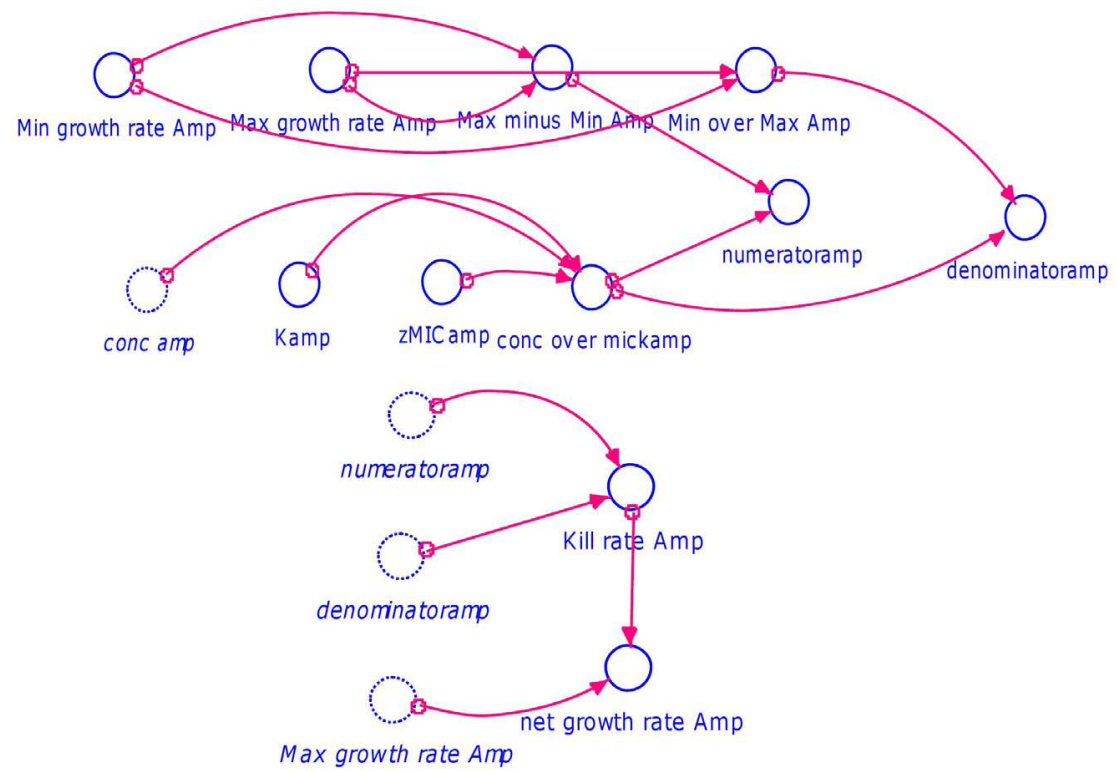

Fig. 2. Sketch of the STELLA ${ }^{\circledR}$ model used to assess the pharmacodynamic effect of the antibiotic Ampicillin.

Bacterial net growth rate against the antibiotic concentration displayed a nonlinear inhibitory PD function with increasing antibiotic concentration. It is assumed that the four PD parameters (MIC, K, minimum growth rate, and maximum growth rate) contribute to the shape of the PD curve. 
It is noticed that concentration dependent antibiotics (Ciprofloxacin, Rifampin, and Streptomycin) showed a sharper decrease in the net growth rate in relation to antibiotic concentration (greater $\mathrm{K}$ value), while time dependent antibiotics (Ampicillin and Tetracycline) showed slower decline with respect to increase in concentration (lower $\mathrm{K}$ values) (Figure 3). Additionally, Figure 4 shows that three antibiotics with the same MIC values and minimum growth rate against a specific microorganism can have different microbiological activity depending on the value of the Hill coefficient $\mathrm{K}$.

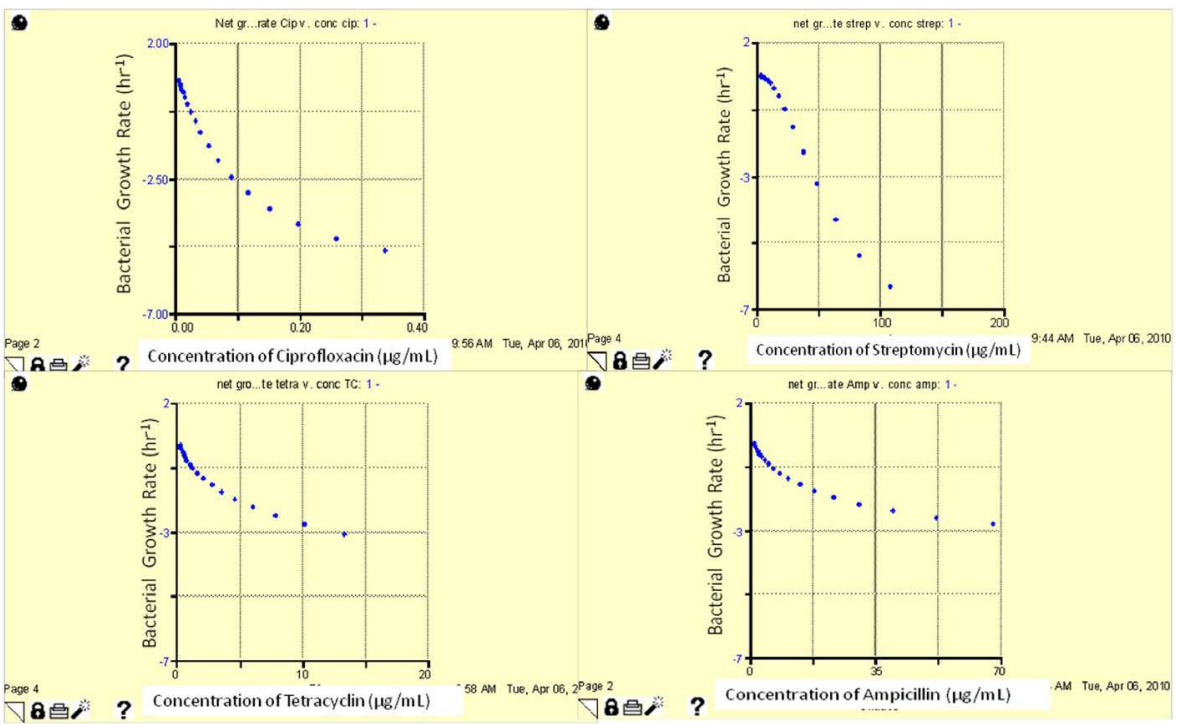

Fig. 3. Effect of antibiotic concentration on bacterial net growth rate

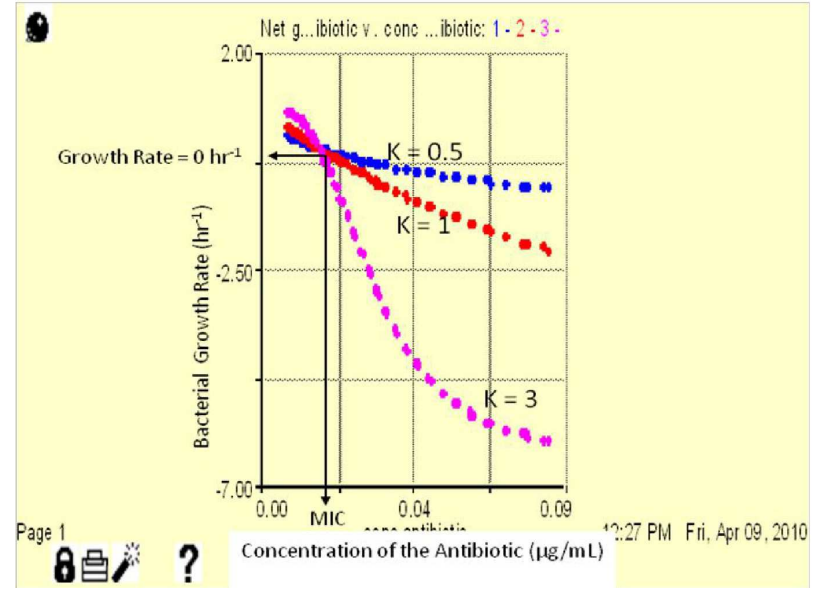

Fig. 4. Pharmacodynamic sensitivity analysis shows the effect of three antibiotics with the same MIC and minimum growth rate on the bacterial net growth rate due to different values of Hill coefficient (K). 


\subsection{PK/PD analysis}

Change in the bacterial density over time was modeled using STELLA ${ }^{\circledR}$ as in figures 5a and b. the model assumes that bacterial growth over time follows an exponential pattern.

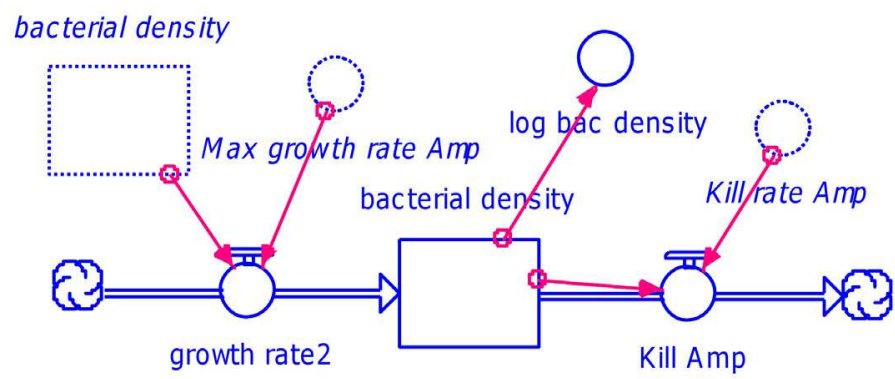

Fig. 5a. STELLA ${ }^{\circledR}$ model constructed to investigate the change in bacterial density over time.

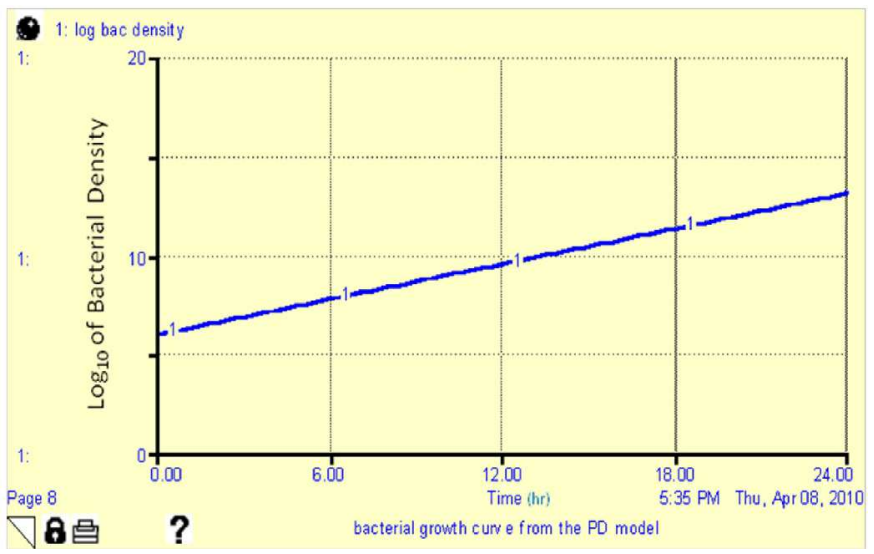

Fig. 5b. Bacterial growth curve from the PD model.

The drug effect was added to the previous model through the inclusion of a first order kill rate. This resulted in a decline in the $\log _{10}$ bacterial density over time. The decline was found to be governed by the magnitude of the PD model parameters. The effect of the Hill coefficient, the minimum growth rate, and the maximum growth rate was investigated for both concentration dependent and time dependent agents. The values of the PD parameters that were used in the sensitivity analysis are reported in Table 2 . The results from the sensitivity analysis are shown in Figures 6, 7 and 8. It is noticed that as $\mathrm{K}$ increases, there is a sharper decline in the bacterial density due to a faster rate of killing. In addition, on the single dose level, ciprofloxacin showed greater rate of killing compared to ampicillin (Figure 6).

Similarly, minimum growth rate achieved under high antibiotic concentration was related to the treatment outcome. Increased minimum growth rate under certain high antibiotic pressure, led to a decreased antibiotic efficacy. This could be attributed to development of resistance. It is also noticed that the effect was greater for concentration dependent antibiotics than the for time dependent agents (Figure 7). 


\begin{tabular}{cccc}
\hline PD parameter & Hill coefficient $(\mathrm{K})$ & $\begin{array}{c}\text { Minimum growth } \\
\text { rate }\left(\mathrm{h}^{-1}\right)\end{array}$ & $\begin{array}{c}\text { Maximum growth } \\
\text { rate }\left(\mathrm{h}^{-1}\right)\end{array}$ \\
\hline Scenario 1 & 0.5 & -3 & 0.75 \\
Scenario 2 & 1 & -6 & 1.2 \\
Scenario 3 & 3 & -9 & 2 \\
\hline
\end{tabular}

Table 2. Pharmacodynamic parameters incorporated in the sensitivity analysis

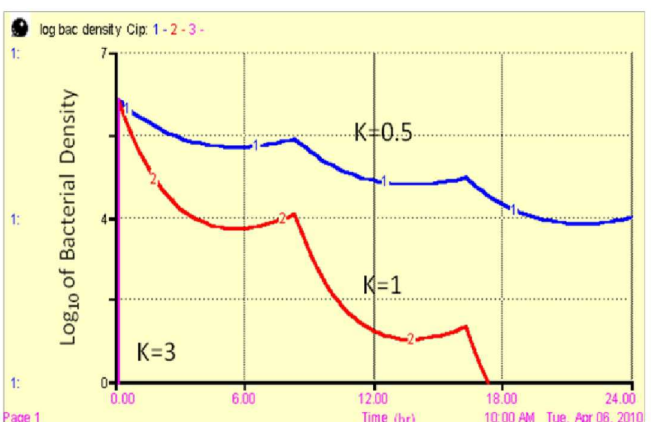

$\nabla 6$ 昌 ?

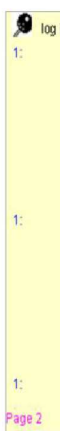

$\nabla \mathbf{8}$ 昌

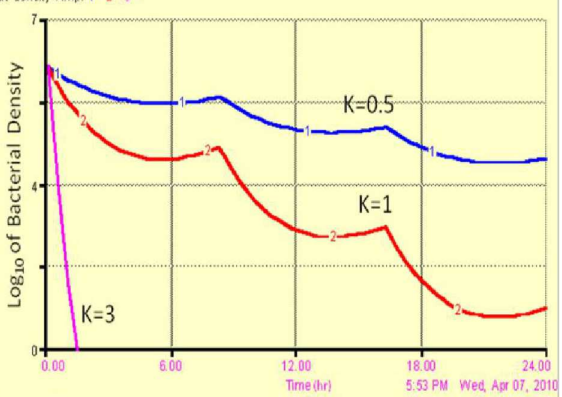

effect of $k$ on the antimicrobial activity of ampicilin

Fig. 6. Effect of the Hill coefficient (K) on the treatment outcomes for both Ciprofloxacin and Ampicillin.

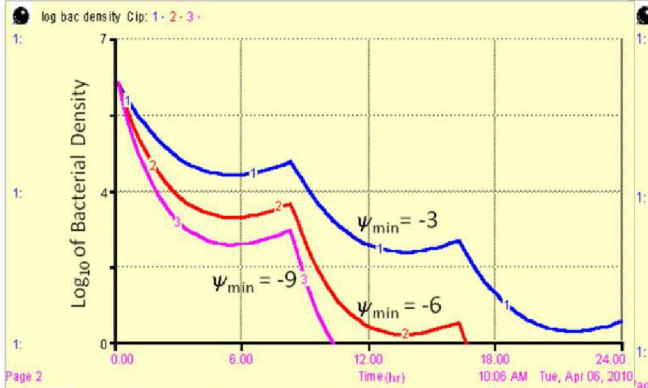

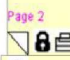
$?$

$9 \log$

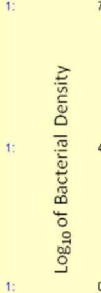

Page 6

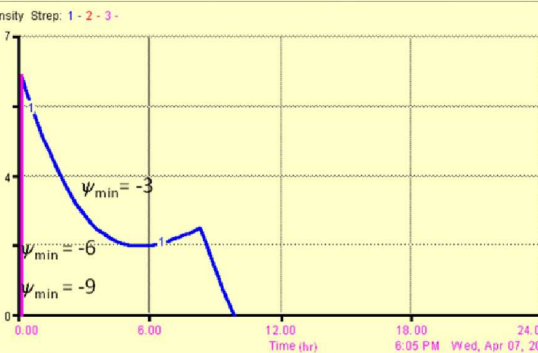

? log bac densty Tetra: 1.2-3.

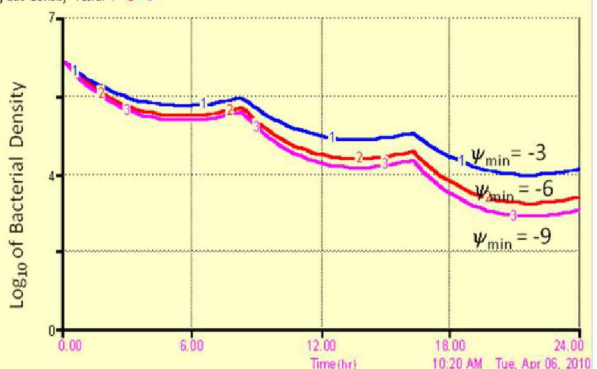

급 log bac densty Anp. 1 - 2-3-

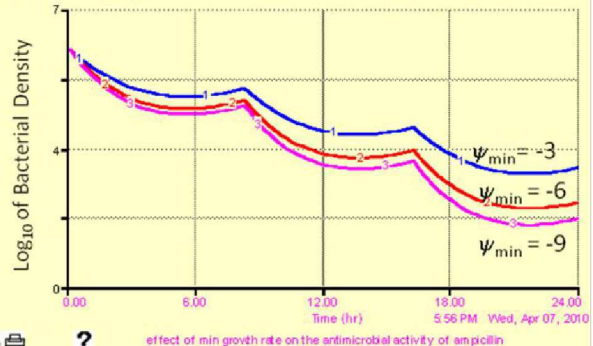

○昌?

Fig. 7. Effect of variation of minimum growth rate achieved under high antibiotic concentration on the treatment outcomes for the investigated antibiotics. 
In addition to the previously mentioned PD parameters, maximum growth rate of the bacteria achieved in the absence of antibiotic was an influential parameter. Change in that parameter could be considered when the effect of the antibiotic on different bacterial strains is a point of interest. Moreover, it could be important for a single microbial strain. For instance, it is known that the growth rate of the bacterial biofilm is reduced compared to the planktonic cultures due to the development of a matrix of extracellular polymeric substance that is referred to as a slime ${ }^{2}$. This will result in reduced penetration of the antibiotics as well as nutrients. Figure 8 augments that by showing that the increase in the maximum growth rate led to a more susceptible micro-organism and a faster kill by the antibiotic.

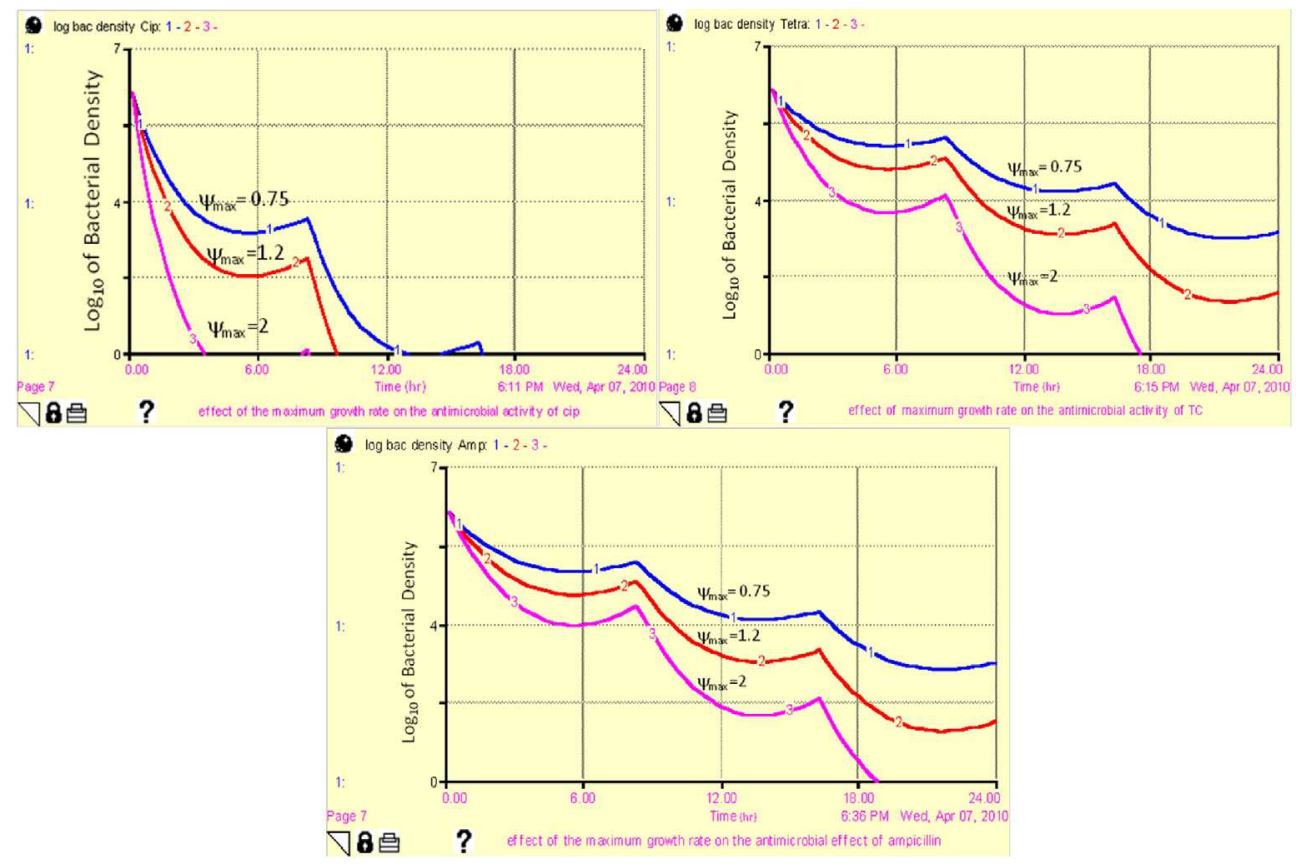

Fig. 8. Effect of variation of maximum growth rate of the microorganism on the treatment outcome with Ciprofloxacin, Tetracycline and Ampicillin.

\section{Development of microbial resistance}

Development of microbial resistance is a major challenge that faces antibiotic treatments and causes reduction in the treatment efficacy. In order to simulate cases of bacterial resistance, MIC values were allowed to vary two and four fold from the original MIC through conducting a zMIC sensitivity analysis (Table 3). The pharmacokinetic profiles of the five antibiotics were simulated using the previously mentioned one compartment disposition model. It is noticed that as the MIC increases, the level of resistance of the microorganism increases, thus an expected reduction in the rate of kill will occur. Under such circumstances, the antibiotic may no longer be effective in eradicating the microorganism as shown in Figure 9 


\begin{tabular}{cccc}
\hline $\begin{array}{c}\text { MIC }(\mu \mathrm{g} / \mathrm{mL}) \\
\text { /antibiotic }\end{array}$ & Scenario 1 & Scenario 2 & Scenario 3 \\
\hline Ciprofloxacin & 0.017 & 0.034 & 0.068 \\
Ampicillin & 3.4 & 6.8 & 13.6 \\
Rifampin & 12 & 24 & 48 \\
Streptomycin & 18.5 & 37 & 74 \\
Tetracycline & 0.67 & 1.34 & 2.68 \\
\hline
\end{tabular}

Table 3. MIC values incorporated in the sensitivity analysis for simulating the development of resistance.
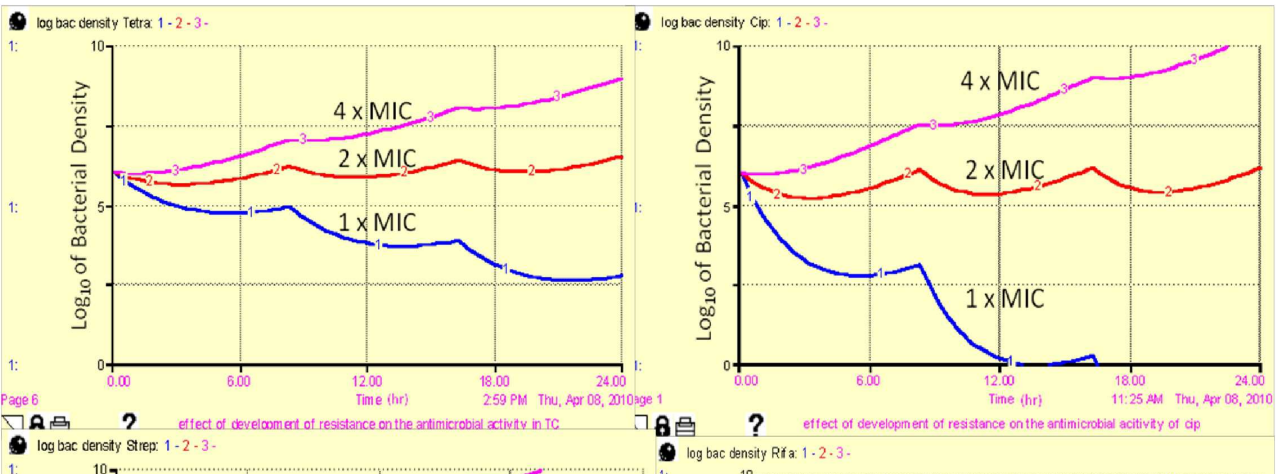

8日 9 ? effect of development of resistance on the antimicrobial acitivity of dip
$9 \log$ bac density Rif a: $1 \cdot 2 \cdot 3$.
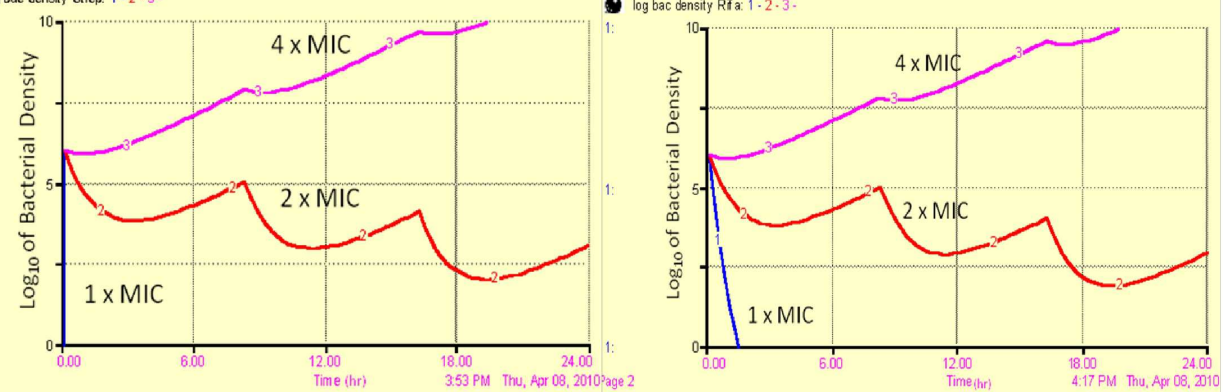

$\nabla \mathbf{8}$

? effect of dev elopment of resist ance on the antimicrobial activity of rif a

Fig. 9. Effect of the development of resistance (simulated through increasing zMIC) on the antimicrobial outcome for the different antibiotics.

\section{Design of treatment strategies}

Each of the five antibiotics was allowed to follow two treatment scenarios to overcome the emergence of the resistant microbe (Figure 10) and the treatment outcome was then evaluated. Treatment modalities included two protocols: the first implemented a method to increase the AUC/MIC by increasing the doses of the antibiotic. The second allowed the percentage of time that the drug concentration stays above the MIC to increase by decreasing the dosing interval while giving small maintenance doses of the antibiotic. Shown in Figure 10 is a representation of the concentration profiles expected from the treatment scenarios and in Figures 11a, b, and c are the simulated treatment outcomes expected from each protocol for three of the antibiotics. 


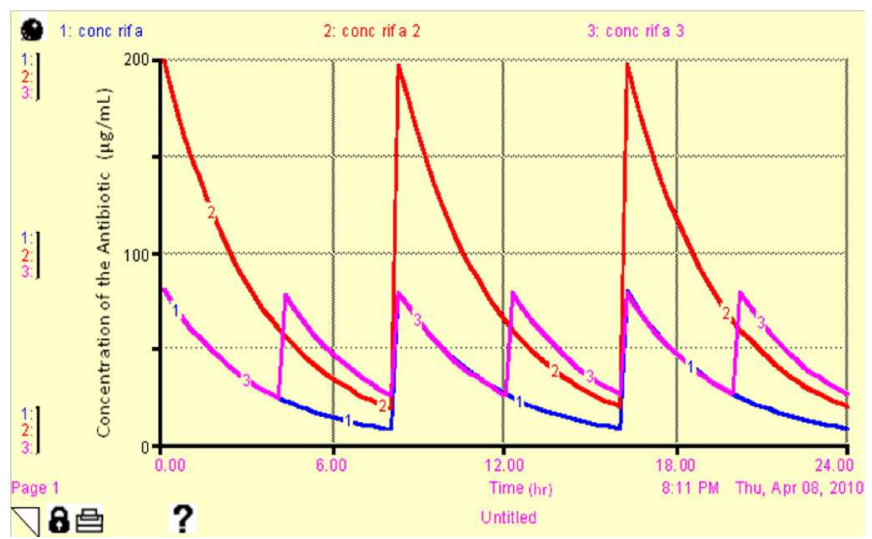

Fig. 10. Simulation of the concentration time profile for the treatment scenarios proposed to overcome resistance. 1. Represents original treatment protocol. 2. Represents increasing AUC $>$ MIC scenario and 3 . Represents the case of increased $\% \mathrm{t}>\mathrm{MIC}$.
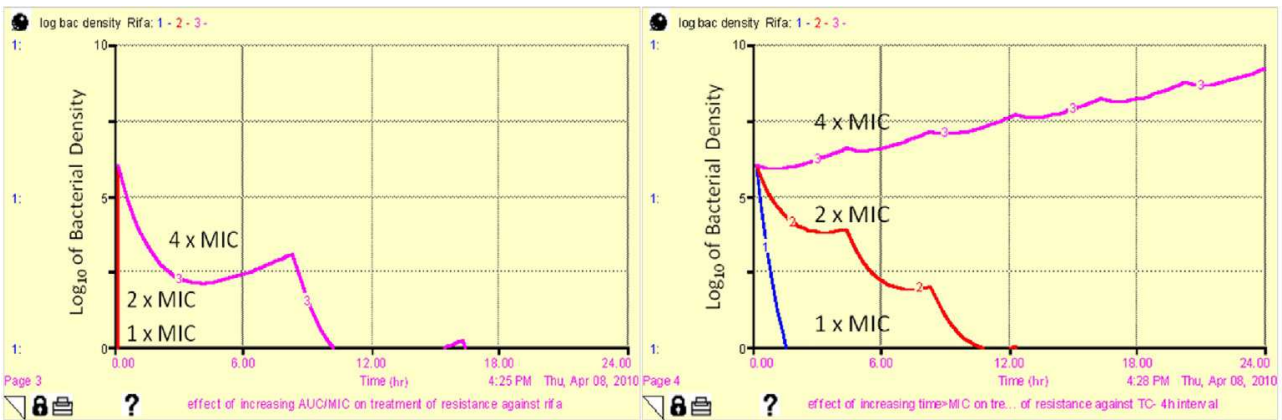

$\nabla \mathbf{8}$ 是

$?$
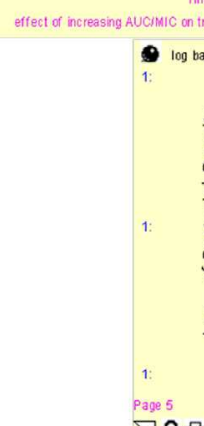

treatment of resistance againgt rit

จ 6 붑

? effect of increasing timey $M C$ on tre of resistance against TC. 4hinterval

$\nabla 6$ 晃

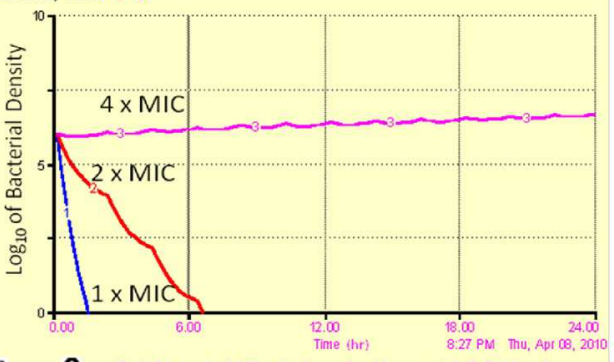

Fig. 11a. Simulation of the target outcome for the two treatment scenarios used against the Rifampin resistance. Upper left: increasing the dose of Rifampin 1.5 folds over 8 hours interval. Upper right: decreasing the dosing interval to 4 hours while maintaining the original dose. Down: decreasing the dosing interval to 2 hours while maintaining the original dose. 

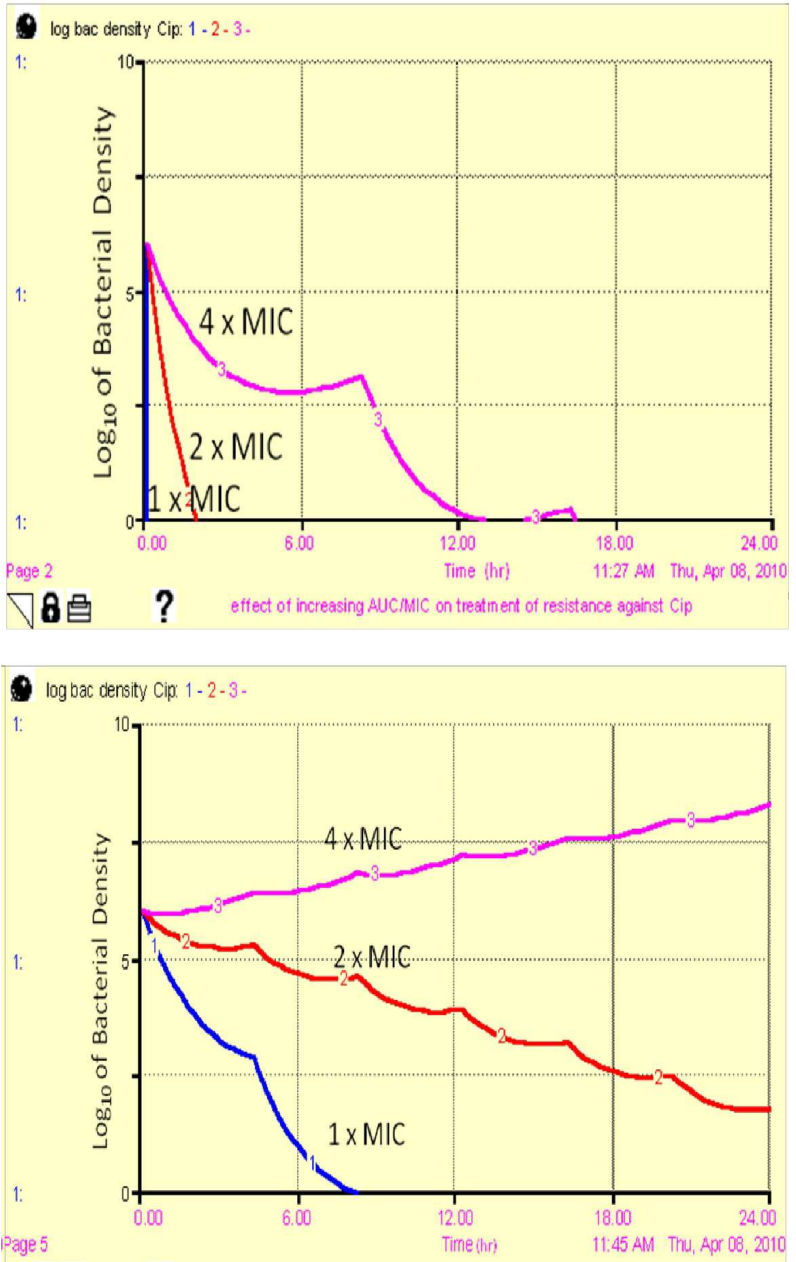

$\nabla 8$ 是 ? effect of treatmert by increasinf \% tMMC on resistance against cip

Fig. 11b. Simulation of the treatment outcomes obtained after implementation of the two treatment scenarios with Ciprofloxacin. Left side curve represents increasing the dose by four times over 8 hours dosing interval. Right side curve represents decreasing the dosing interval by 4 hours while maintaining the original dose.

Rifampin is known to be a concentration-dependent antibiotic. Increasing the exposure to the drug above the MIC (by 1.5 fold increase in the dose) improved the treatment outcome, yet increasing the percentage of time relative to the dosing interval (tau) that the drug concentration was above the MIC- by decreasing tau from 8 hours to 4 and 2 hours simultaneously- did not achieve the target outcome even when the dosing interval decreased to 2 hours. Ciprofloxacin- a fluoroquinolone known to be a concentration dependent antibiotic- displayed similar results to Rifampin upon increasing exposure above MIC (four fold increase in the dose) compared to $\mathrm{t}>\mathrm{MIC}$ (reducing the dosing interval to 2 hours). 

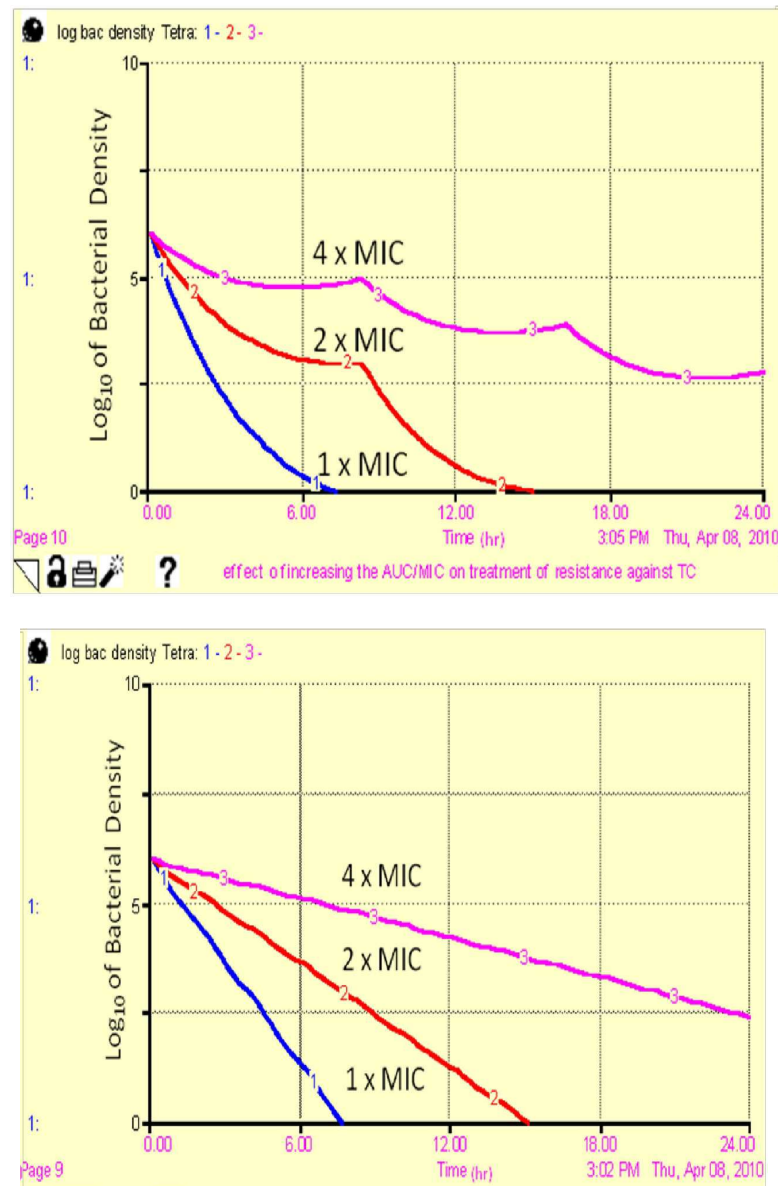

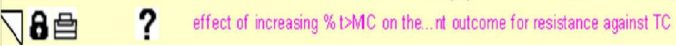

Fig. 11c. Simulation of the treatment outcomes achieved after increasing Tetracycline dose (four folds over 8 hours dosing interval, left side curve), or decreasing the dosing interval (2 hours, while maintaining original dose, right side curve).

On the other hand, the efficacy of Tetracycline is known to be time dependent. Therefore, increasing the percent of time the drug stays above MIC relative to the dosing interval seems to be more relevant than increasing the exposure above the MIC. Surprisingly, the two scenarios have achieved nearly the same treatment outcome for microbial resistance against Tetracycline. Both treatment scenarios succeeded to achieve the target outcome of three $\log _{10}$ reduction in bacterial density by the end of the antibiotic treatment.

\section{Discussion}

This study addressed the complex relationship between the exposure to antibiotics and the growth and death rates of bacteria. PK/PD simulations using STELLA ${ }^{\circledR}$ showed that 
although MIC is an important PD parameter, it is not the only parameter that governs the interaction of the antibiotic and the bacteria. Other PD parameters should additionally be taken into consideration when designing antibiotic dosage regimens. For instance, Figure 4 showed that three antibiotics with the same MIC (the concentration corresponding to a net growth of zero) could show different microbiological activity depending on the magnitude of the Hill coefficient (K). Other PD parameters to be considered are the minimum growth rate and the maximum growth rate, which can capture effects that cannot be accounted for by solely considering the MIC and can importantly affect the therapeutic outcomes.

Pharmacodynamic simulations of the antibiotics concentration against net bacterial growth showed that the shape of the sigmoidal functions differs among antibiotics from different classes with some of them showing greater sensitivity to changing concentrations (Ciprofloxacin and Streptomycin) than others (Tetracycline and Ampicillin). The shape of the pharmacodynamic function is determined by the values of PD parameters displayed in Table 2.

Additionally, using a STELLA ${ }^{\circledR}$ model for the change in bacterial density over time, it was found that the parameters that govern the PD function have a profound effect on the microbiological activity. The Hill coefficient influences the sensitivity of the bacteria to the change in the antibiotic concentration as was shown in Figure 4. Assuming a PK model in which the drug reaches steady state form the beginning of the treatment, a concentration that is above the MIC most of the time, and all other parameters are equal, the simulation predicts that antibiotics with a high Hill coefficient are more effective than those with a low value of the coefficient.

Considering the minimum bacterial net growth rate at high antibiotic concentrations, the simulation predicts that antibiotics that induced lower values of minimum bacterial net growth rate are more effective than those with higher values. Increase in the minimum bacterial net growth rate could be atrributed to the development of resistance or to the utilization of ineffective therapy against a specific bacterial strain.

Maximum growth rate in the absence of antibiotic is another important parameter to consider in the design of antibiotic treatment. That PD parameter is subject to change when treating different micro-organisms or if the nature of infection by the same microbe has been changed. Regarding the later condition, biofilm infection could be considered as a change in the nature of the infection by the same micro-organism ${ }^{5}$. It is well-known that biofilm is a type of persistent infections that is developed in chronic disease statuses and is characterized by a slower rate of growth compared to acute infections ${ }^{2}$. This phenomenon is also observed in the in vitro systems- where the planktonic cultured bacteria exhibit a faster rate of growth compared to the biofilm cultured ones ${ }^{6}$. Therefore, increasing the maximum growth rate in the sensitivity analysis has led to a faster kill by different antibiotics due to the greater exposure of the bacteria to the antibiotic compared to the slowly growing biofilm infection.

Interestingly enough, estimates of the parameter $\mathrm{K}$ was higher for antibiotics that are concentration dependent (Ciprofloxacin, Rifampin, and Streptomycin) compared to those that are time dependent (Ampicillin and Tetracycline) which means that the formers are 
characterized by a steeper pharmacodynamic function. The steeper the function, the more dramatically the bacterial killing is sensitive to the change in antibiotic concentration above the MIC. Therefore, it could be a reasonable assumption that for antibiotics that have grater $\mathrm{K}$, increasing the concentration above MIC (AUC/MIC) is the most effective strategy to improve the treatment outcomes. This hypothesis was fully augmented by the PK/PD simulation shown in both Figures $11 \mathrm{a}$ and $\mathrm{b}$. Increasing the $\% \mathrm{t}>\mathrm{MIC}$ for Rifampin by reducing the dosing interval to 4 hours and even 2 hours did not achieve the target outcome for the resistant strain as did the increase in the AUC/MIC. Furthermore, Ciprofloxacin achieved the target therapeutic outcome only when the dose increased allowing AUC>MIC to increase. It is worth mentioning that the magnitude of the dose increase for concentration dependent antibiotics appears to be related to the PD parameter $\mathrm{K}$ (the Hill coefficient); since Rifampin- has a K value of 2.5 compared to 1.1 for Ciprofloxacin- has achieved the target therapeutic outcome upon 1.5 fold increase in the dose compared to a four folds increase for Ciprofloxacin. In another way, one can conclude that as $\mathrm{K}$ increases, as the antibiotic efficacy is more sensitive to small changes in concentration.

On the other hand, it was assumed that lower values of $\mathrm{K}$ could be associated with time dependence of the antibiotics. This assumption is not fully supported by the current simulation, since for Tetracycline $(\mathrm{k}=0.61)$ both scenarios of increasing the AUC/MIC and $\% \mathrm{t}>\mathrm{MIC}$ worked well and achieved comparable therapeutic outcomes. It is worth mentioning, however, that the maintenance dose used in the former scenario $(12.08 \mu \mathrm{g}$ every 8 hours) was four times higher than that used in the later scenario ( $2.95 \mu \mathrm{g}$ every 2 hours). Although it is known that longer dosing intervals are more convenient and can improve patient adherence to therapy, drug toxicity is an important consideration as well. In case where toxicity is expected from higher doses of a time-dependent antibiotic, the strategy of dividing the doses into smaller fractions for shorter dosing intervals may be considered.

Finally, it should be noted that this model may suffer some limitations due to many causes. The model assumes that the kill rate increases with increasing antibiotic concentration, however in reality, the development of an adaptive resistance- a mechanism by which the bacteria becomes increasingly refractory to the antibiotic treatment- may lead to the decrease in kill rate with time and therefore limit such assumptions. Moreover, the constitutive and inducible immune defense of the human body contributes to the efficacy of the antibiotic treatment protocols in a way that could not be fully represented by the current $\mathrm{PK} / \mathrm{PD}$ analysis under in vitro conditions.

\section{References}

[1] Roland R. Regoes, Camilla Wiuff, Renata M. Zappala, Kim N. Garner, Fernando Baquero, and Bruce R. Levin. Pharmacodynamic Functions: a Multiparameter Approach to the Design of Antibiotic Treatment Regimens. Antimicrobial agents and chemotherapy. October 2004, 48: 3670-3676 
[2] Hall-Stoodley L, Costerton JW, Stoodley P. Bacterial biofilms: from the natural environment to infectious diseases. Nature Reviews. Microbiology. February 2004, 2 (2): 95-108.

[3] Marc H. Scheetz, Kristin M. Hurt, Gary A. Noskin and Catherine M. Oliphant. Applying antimicrobial pharmacodynamics to resistant gram-negative pathogens. Am J Health-Syst Pharm. July 2006, 63: 1346-1360

[4] Julia Gloede, Christian Scheerans, Hartmut Derendorf and Charlotte Kloft. In vitro pharmacodynamic models to determine the effect of antibacterial drugs. J Antimicrob Chemother. 2010, 65: 186-201

[5] Daniel J Hassett, Thomas R Korfhagen, Randall T Irvin, Michael J Schurr, Karin Sauer, Gee W Lau, Mark D Sutton, Hongwei Yu and Niels Hoiby. Pseudomonas aeruginosa biofilm infections in cystic fibrosis: insights into pathogenic processes and treatment strategies. Expert Opin Ther Targets. 2010, 14:117-30

[6] Thien-Fah C. Mah and George A. O'Toole. Mechanisms of biofilm resistance to antimicrobial agents. Trends in Microbiology. 2001, 9:34-39 


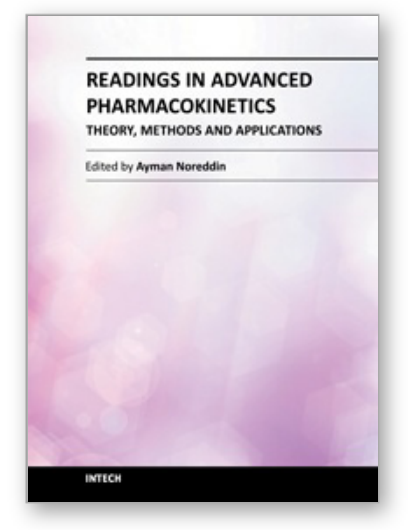

\section{Readings in Advanced Pharmacokinetics - Theory, Methods and Applications}

Edited by Dr. Ayman Noreddin

ISBN 978-953-51-0533-6

Hard cover, 378 pages

Publisher InTech

Published online 20, April, 2012

Published in print edition April, 2012

This book, "Readings in Advanced Pharmacokinetics - Theory, Methods and Applications", covers up to date information and practical topics related to the study of drug pharmacokinetics in humans and in animals. The book is designed to offer scientists, clinicians and researchers a choice to logically build their knowledge in pharmacokinetics from basic concepts to advanced applications. This book is organized into two sections. The first section discusses advanced theories that include a wide range of topics; from bioequivalence studies, pharmacogenomics in relation to pharmacokinetics, computer based simulation concepts to drug interactions of herbal medicines and veterinary pharmacokinetics. The second section advances theory to practice offering several examples of methods and applications in advanced pharmacokinetics.

\section{How to reference}

In order to correctly reference this scholarly work, feel free to copy and paste the following:

Ghada F. Ahmed and Ayman M. Noreddin (2012). Application of Pharmacokinetics/Pharmacodynamics (PK/PD) in Designing Effective Antibiotic Treatment Regimens, Readings in Advanced Pharmacokinetics Theory, Methods and Applications, Dr. Ayman Noreddin (Ed.), ISBN: 978-953-51-0533-6, InTech, Available from: http://www.intechopen.com/books/readings-in-advanced-pharmacokinetics-theory-methods-andapplications/application-of-pharmacokinetics-pharmacodynamics-pk-pd-in-designing-effective-antibiotictreatment-r

\section{INTECH}

open science | open minds

\section{InTech Europe}

University Campus STeP Ri

Slavka Krautzeka 83/A

51000 Rijeka, Croatia

Phone: +385 (51) 770447

Fax: +385 (51) 686166

www.intechopen.com

\section{InTech China}

Unit 405, Office Block, Hotel Equatorial Shanghai

No.65, Yan An Road (West), Shanghai, 200040, China

中国上海市延安西路65号上海国际贵都大饭店办公楼 405 单元

Phone: +86-21-62489820

Fax: $+86-21-62489821$ 
(C) 2012 The Author(s). Licensee IntechOpen. This is an open access article distributed under the terms of the Creative Commons Attribution 3.0 License, which permits unrestricted use, distribution, and reproduction in any medium, provided the original work is properly cited. 